\title{
Relaxin is a potent renal vasodilator in conscious rats
}

\author{
Lee A. Danielson, ${ }^{1}$ O. David Sherwood, ${ }^{2}$ and Kirk P. Conrad ${ }^{3}$
}

${ }^{1}$ Department of Pathology, University of New Mexico School of Medicine, Albuquerque, New Mexico 87131, USA

${ }^{2}$ Department of Molecular and Integrative Physiology and College of Medicine, University of Illinois at Urbana-Champaign, Urbana, Illinois 61801, USA

${ }^{3}$ Departments of Obstetrics, Gynecology, and Reproductive Sciences, and of Cell Biology and Physiology, University of Pittsburgh and Magee-Womens Research Institute, Pittsburgh, Pennsylvania 15213, USA

Address correspondence to: Kirk P. Conrad, Magee-Womens Research Institute, 204 Craft Avenue, Pittsburgh, Pennsylvania 15213, USA. Phone: (412) 641-6019; Fax: (412) 641-1503; E-mail: rsikpc@mail.magee.edu

Portions of this work were published in abstract form (1998. J. Soc. Gynecol. Invest. 5:146A; 1998. J. Am. Soc. Nephrol. 9:336A).

Received for publication October 27, 1998, and accepted in revised form January 4, 1999.

The kidneys and other nonreproductive organs vasodilate during early gestation; however, the "pregnancy hormones" responsible for the profound vasodilation of the renal circulation during pregnancy are unknown. We hypothesized that the ovarian hormone relaxin (RLX) contributes. Therefore, we tested whether the administration of RLX elicits renal vasodilation and hyperfiltration in conscious adult, intact female rats. After several days of treatment with either purified porcine RLX or recombinant human RLX 2 (rhRLX), effective renal plasma flow (ERPF) and glomerular filtration rate (GFR) increased by $20 \%-40 \%$. Comparable renal vasodilation and hyperfiltration was also observed in ovariectomized rats, suggesting that estrogen and progesterone are unnecessary for the renal response to rhRLX. The nitric oxide synthase inhibitor $\mathrm{N}^{\omega}$-nitro-L-arginine methyl ester completely abrogated the increase in ERPF and GFR elicited by chronic administration of purified porcine RLX. In contrast, the renal vasoconstrictory response to angiotensin II was attenuated by the RLX treatment. Short-term infusion of purified porcine RLX to conscious rats over several hours failed to increase ERPF and GFR. Plasma osmolality was consistently reduced by the chronic administration of both RLX preparations. In conclusion, the renal and osmoregulatory effects of chronic RLX administration to conscious rats resemble the physiological changes of pregnancy in several respects: (a) marked increases in ERPF and GFR with a mediatory role for nitric oxide; $(b)$ attenuation of the renal circulatory response to angiotensin II; and $(c)$ reduction in plasma osmolality.

J. Clin. Invest. 103:525-533 (1999).

\section{Introduction}

The hormone relaxin (RLX) has a molecular weight of approximately 5,000-6,000 and is a member of the insulin-growth factor family. In humans and rats, the ovary is the major, if not sole, source of circulating RLX. In pregnant rats, RLX promotes growth and softening of the cervix and vagina, promotes growth of the mammary nipples, and inhibits spontaneous uterine contractility (reviewed in ref. 1).

During early pregnancy in humans, rats, and other mammals, vasodilation of nonreproductive organs leads to a marked decline in total peripheral vascular resistance (2). The kidneys contribute to this fall in peripheral resistance; a nadir in renal vascular resistance and a reciprocal peak in renal blood flow and glomerular filtration rate of $40 \%-80 \%$ above nonpregnant levels are reached by the end of the first trimester in women (reviewed in ref. 3). Maximal changes, albeit of a lesser magnitude (20\%-40\% from nonpregnant levels), are observed in the renal circulation of conscious rats by gestational day 12 (3).

The "pregnancy hormones" that cause renal vasodilation and hyperfiltration have not been identified. For several reasons, we hypothesized that RLX contributes. First, the plasma concentration of RLX increases rapidly after conception in women (reviewed in ref. 1), corresponding with the marked increase in both glomerular filtration rate (GFR) and effective renal plasma flow (ERPF) during the first trimester (3). The stimulus for secretion of RLX by the corpus luteum is human chorionic gonadotrophin (hCG), which rises in parallel (1). Second, circulating RLX also increases during the luteal phase of the menstrual cycle $(1,4-6)$, coinciding with a transient $20 \%$ increase in GFR and ERPF (7-12). Third, the gestational rise in RLX corresponds with another early physiological adaptation in human pregnancy, namely, osmoregulatory changes (13). Chronic administration of recombinant human RLX 2 (rhRLX) to ovariectomized rats reduced plasma osmolality and the osmotic threshold for arginine vasopressin release to levels comparable to the gravid condition (14). Fourth, the osmoregulatory changes of pregnancy were mimicked by administering hCG to women in the luteal phase of the menstrual cycle and intact female rats, but not to men or ovariectomized rats, suggesting the intermediary role of an ovarian hormone (15-17). Associated with the osmoregulatory changes observed in women after administration of hCG was a $15 \%-20 \%$ rise in GFR, which again was not observed in men administered the hormone (Davison, J.M., personal communication). Fifth, although renal vasodilation and hyperfiltration are observed in gravid rats as early as day 5 of gestation, before detectable increases in ovarian and plasma RLX occur, there is a marked jump in GFR and ERPF between gestational days 8 and 12 (18), when ovarian and circulating 
RLX surge (19). Conceivably, the early increase in GFR and ERPF in pregnant rats - and the comparable renal changes in pseudopregnant rats $(20,21)$ - stem from circulating RLX, which is below the sensitivity of assay detection. To summarize, circumstantial evidence implicates RLX in the control of renal circulation during pregnancy.

In this study, the effect of administered purified porcine and rhRLX on blood pressure and renal function was tested in chronically instrumented conscious rats. RLX proved to be a potent renal vasodilator. Because we showed previously that gestational renal vasodilation and hyperfiltration in conscious rats are mediated by nitric oxide (NO) via endothelin (ET) and the endothelial $\mathrm{ET}_{\mathrm{B}}$ receptor subtype (22-24), we also investigated whether NO mediated the renal vasodilatory response to RLX.

\section{Methods}

Animal preparation. Long-Evans female rats 10-14 weeks old were purchased from Harlan Sprague-Dawley (Frederick, Maryland, USA). They were fed PROLAB RMH 2000 diet containing 0.48\% sodium (PME Feeds Inc., St. Louis, Missouri, USA) and were provided water ad libitum. To prepare the rats for experimental procedures, they were trained for several hours in a Plexiglas restraining cage (Braintree Scientific Co., Braintree, Massachusetts, USA) on at least five different occasions before surgical intervention. These cages afforded sufficient space for grooming of the face and front paws while preventing the rat from turning around. Thus, accurate timed urine collections and blood samplings were made possible from the chronically implanted bladder and vascular catheters, respectively. Rats failing to habituate to the cage were eliminated from the study $(<1 \%)$. All animal procedures were approved by the Institutional Animal Care and Use Committee of the Magee-Womens Research Institute.

The details of the surgical procedures have been described previously $(18,22)$. Briefly, using ketamine $(6 \mathrm{mg} / 100 \mathrm{~g}$ body weight) and pentobarbital sodium ( $2.1 \mathrm{mg} / 100 \mathrm{~g}$ body weight) anesthesia, Tygon catheters were implanted in the abdominal aorta and inferior vena cava via the femoral artery and vein, respectively. The bladder catheter, a silastic-covered stainless steel cannula, was sewn into the urinary bladder with a purse-string suture and exteriorized through the ventral abdominal wall. This catheter was then plugged, allowing the animal to urinate through the urethra while in her home cage. In the ovariectomized and sham-ovariectomized animals, the ovaries were first ligated and then excised, or briefly manipulated, respectively, via a small incision made in the lateral abdominal wall immediately after the insertion of vascular and bladder catheters. All surgeries were conducted using aseptic technique. At least a 7-day recovery period was allowed before experimentation.

For chronic infusion of RLX, an osmotic minipump was inserted subcutaneously in the back of the animal under ether anesthesia. After shaving and cleaning the skin with alcohol and betadine, a small incision was made and the minipump containing either rhRLX, purified porcine RLX, or vehicle was inserted. Then the incision was closed with surgical staples. The model 2001 and 2ML1 osmotic minipumps (Alza Co., Palo Alto, California, USA) were used for the purified porcine RLX and rhRLX, respectively.

\section{Experimental protocols}

Influence of chronic infusion of purified porcine $R L X, r b R L X$, or vehicle on renal function in intact female rats. At the beginning of each experiment, immediately after opening the arterial catheter and before infusion of fluids, $100 \mu \mathrm{l}$ of blood was collected into a heparinized tube. After centrifugation to separate blood cells from plasma, the latter was frozen at $-20^{\circ} \mathrm{C}$ until it was assayed for osmolality. Then three $30-\mathrm{min}$ baseline urine collections with midpoint blood samples and continuous recording of mean arterial pressure (MAP) were made on one or two occasions, separated by at least $48 \mathrm{~h}$, to accurately measure control MAP, GFR, ERPF, and effective renal vascular resistance (ERVR) (MAP/ERPF). The hematocrit was also routinely determined on these midpoint blood samples. The renal clearances of inulin (IN) and para-aminohippurate (PAH) were used to assess GFR and ERPF, respectively. The femoral arterial catheter was connected to a Statham pressure transducer (Gould P23 ID; Statham Instruments, Hato Rey, Puerto Rico) and a Gould Universal amplifier for measurement of MAP, which was displayed on a Gould 5900 Series Signal Conditioner Cage and TA11 chart recorder. Next, an osmotic minipump containing purified porcine RLX (4 $\mu \mathrm{g} / \mathrm{h} ; n=7)$, rhRLX $(4 \mu \mathrm{g} / \mathrm{h} ; n=5)$, or vehicle ( $n$ $=4$ ) was implanted. MAP, GFR, ERPF, and ERVR were assessed on days 2 and 5 of the infusion, and 5-12 days after depletion of the 7-day minipump. Details of our methodologies for the assessment of GFR and ERPF in chronically instrumented, conscious rats, including the measurement of IN and PAH in both plasma and urine, have been published previously $(18,22)$. Circulating RLX concentrations of $20-40 \mathrm{ng} / \mathrm{ml}$ have been measured in rats of 12-14 gestational days (19). Based on previous work $(14,25)$, an infusion rate of $4 \mu \mathrm{g} / \mathrm{h}$ for purified porcine RLX or rhRLX would be expected to produce plasma levels of $20-40 \mathrm{ng} / \mathrm{ml}$. In fact, we reached this target level; see Results.

Influence of chronic infusion of rhRLX on renal function in ovariectomized or sham-ovariectomized rats. Six female rats each were subjected either to ovariectomy or sham ovariectomy immediately after the implantation of vascular and bladder catheters. Seven days later, renal function and MAP were assessed before the insertion of osmotic minipumps containing rhRLX. On day 5 of the RLX infusion $(4 \mu \mathrm{g} / \mathrm{h})$, renal function and MAP were again determined.

Acute infusion of $N^{\omega}$-nitro-L-arginine methyl ester or angiotensin II during chronic infusion of purified porcine RLX. At least 7 days after the implantation of vascular and bladder catheters, osmotic minipumps containing either porcine RLX $(4 \mu \mathrm{g} / \mathrm{h})$ or vehicle (Ringer's solution) were implanted in intact female rats $(n=7$ and 6, respectively). Renal function and MAP were measured on day 5 of RLX infusion (three 30-min urine and midpoint blood collections). Next, an infusion of $N^{\omega}$-nitro-L-arginine methyl ester (L-NAME) $(2 \mu \mathrm{g} / \mathrm{min}$; a substrate competitive inhibitor of NO synthase) was administered intravenously by infusion pump (Model 200; KD Scientific, Boston, Massachusetts). Four 1-h urine collections with midpoint blood samples were collected for the assessment of GFR, ERPF, and ERVR during the L-NAME administration. Identical procedures were performed on additional animals chronically administered porcine RLX $(n=5)$ or vehicle $(n=5)$, except that angiotensin II (ANG II) $(3 \mathrm{ng} / \mathrm{min})$ was infused rather than L-NAME. Finally, another four age-matched control rats were administered Ringer's solution instead of either L-NAME or ANG II on day 5 of porcine RLX infusion.

Acute administration of purified porcine $R L X$. Renal function and MAP were measured in four intact female rats during acute infusion of purified porcine RLX. Three 30-min baseline urine collections were obtained with midpoint blood samples to accurately determine control GFR, ERPF, and ERVR. Then a bolus of $2 \mu \mathrm{g}$ purified porcine RLX in $0.5 \mathrm{ml}$ total volume was given over $5 \mathrm{~min}$ into the femoral venous catheter to fill the extracellular water compartment and reach a target concentration of $20-40 \mathrm{ng} / \mathrm{ml}$. Immediately thereafter, porcine RLX was administered at $4 \mu \mathrm{g} / \mathrm{h}$ by infusion pump, and four 1-h urine collections with midpoint blood samples were made during the RLX infusion.

Metabolic cage studies. Six rats were individually housed in Nalgene metabolism cages (Rodent Metabolism Cages for 150-300 g rats; VWR Scientific, Bridgeport, New Jersey, USA). Water and 
food were provided ad libitum. After 5-7 days of habituation, two baseline 24-h urine collections were obtained. Then an osmotic minipump containing purified porcine RLX was implanted $(4 \mu \mathrm{g} / \mathrm{h})$. Additional 24-h urine collections were made on days 2 and 5 of RLX infusion and on days 4, 12, and 25 after exhaustion of the 7-day minipump. Food and water intake, as well as urinary flow rate were measured by gravimetric technique. The urinary excretion of sodium, cGMP, and nitrate + nitrite $\left(\mathrm{NO}_{\mathrm{x}}\right)$ were also determined. The measurements made during the two baseline collections were averaged, as were the measurements made during the three post-RLX collections.

Analytic techniques. Plasma osmolality was measured using a freezing-point depression instrumentation osmometer (Model $3 \mathrm{MO}$; Advanced Instruments, Needham Heights, Massachusetts, USA). Plasma and urine IN and PAH were assayed by standard techniques as reported previously $(18,22)$. Urinary sodium concentration was measured by ion selective electrode (SodiumPotassium Chemistry Module; Beckman Instruments Inc., Brea, California, USA). Urinary cGMP was determined by specific radioimmunoassay as described previously (26). Urinary $\mathrm{NO}_{X}$ was measured by reduction of nitrate to nitrite, the latter determined by the Griess reaction, which produces a colorimetric product measured at $540 \mathrm{~nm}$ (Nitrate/Nitrite Colorimetric Assay Kit; Cayman Chemical, Ann Arbor, Michigan, USA).

One milliliter of blood was obtained from the rats administered with rhRLX for 5 days at the end of the renal function measurements. The levels of rhRLX in serum were then measured in a quantitative sandwich immunoassay (27). Briefly, wells of a 96-well microtiter plate (Maxisorp Immunomodules; Nalge Nunc International, Naperville, Illinois, USA) were coated overnight with affinity-purified anti-rhRLX rabbit polyclonal antibody. Sera were diluted in PBS containing Tween-20, Thimerosal, BSA (Sigma Chemical Co., St. Louis, Missouri, USA), and normal goat IgG (Organon Teknika-Cappel, Durham, North Carolina, USA), and $100 \mu \mathrm{l}$ was added to wells in duplicate. After an overnight incubation at $4^{\circ} \mathrm{C}$, the wells were washed and $100 \mu \mathrm{l}$ of affinity-purified, peroxidase-conjugated anti-rhRLX rabbit polyclonal antibody was added to each well. After an appropriate incubation period at room temperature, the wells were washed again, and $100 \mu \mathrm{l}$ of a tetramethylbenzidine solution was added to each well. After color development, the reaction was stopped, absorbances at 450/630 nm were measured, and RLX concentrations in the sera were determined by entering data into a four-parameter logistic curve-fitting program. The assay has been validated for use with murine serum, shows no detectable cross-reactivity with natural murine RLX, and has a lower detection limit of $20 \mathrm{pg} / \mathrm{ml}$.

Preparation of drugs. PAH and IN were freshly prepared on the morning of the experiment using Ringer's solution as diluent. IN, characteristically insoluble at ambient temperature, was prepared for infusion by heating a $15-\mathrm{ml}$ aliquot in a boiling water bath for $10 \mathrm{~min}$. When diluted in Ringer's solution and mixed with $\mathrm{PAH}$, it remained in solution throughout the experiment. L-NAME (Sigma Chemical Co.) was also prepared in Ringer's solution within $1 \mathrm{~h}$ of use. ANG II (5-ILE All or Hypertensin II; Sigma Chemical Co.) was prepared from a stock solution in $5 \%$ dextrose $(100 \mu \mathrm{g} / \mathrm{ml})$ that was frozen in aliquots at $-20^{\circ} \mathrm{C}$. Final dilutions were made in Ringer's solution immediately before infusion. For chronic infusion by osmotic minipump, rhRLX (Connectics, Palo Alto, California, USA) was prepared from a stock $1.5 \mathrm{mg} / \mathrm{ml}$ solution in $20 \mathrm{mM}$ sodium acetate ( $\mathrm{pH}$ 5.0) and diluted accordingly in the same buffer, and the lyophilized, purified porcine RLX (28) was dissolved in Ringer's solution. For acute infusion, the lyophilized purified porcine RLX was prepared daily immediately before use in Ringer's solution containing $0.01 \%$ rat albumin (Cappel Research Products, Durham, North Carolina, USA).

Statistical analysis. Data are presented as mean \pm SEM. Most data were analyzed using repeated-measures mixed models with treatment group and time as fixed effects. If significant main effects or interactions were observed, then Dunnett's test was used to compare least-squares means for baseline values with all subsequent time periods. Least-squares means for rats that had been administered vehicle and recombinant human and purified porcine RLX were compared using Scheffe's procedure for multiple comparisons. For Table 5, one-factor repeated-measures ANOVA was used, and group means were compared by Orthogonal Contrasts. $P<0.05$ was considered statistically significant (29).

\section{Results}

Chronic infusion of rhRLX, purified porcine $R L X$, or vebicle. The time-control experiments (Fig. 1, $a-d$ ) using either the vehicle for rhRLX (20 mM sodium acetate, $\mathrm{pH}$ 5.0; $n$ $=2$ ) or the vehicle for porcine RLX (Ringer's solution; $n$ $=2$ rats) showed relative stability of MAP, GFR, ERPF, and ERVR over the approximately two-week study period $(P=$ not significant [ns] by ANOVA). Because the results obtained with the two different vehicle preparations were comparable, they were combined. While chronic infusion of purified porcine RLX at $4 \mu \mathrm{g} / \mathrm{h}$ did not significantly change MAP, there was a marked increase in GFR and ERPF, as well as a reciprocal reduction in ERVR on both days 2 and 5 of administration $(P$ $<0.05$ vs. baseline and vehicle). Similar results were obtained for rhRLX, except that significance was not reached until day 5 of infusion.

Chronic infusion of rhRLX in ovariectomized or sham-ovariectomized rats. The profound rise in GFR and ERPF, as well as fall in ERVR, observed on day 5 of RLX infusion were comparable in ovariectomized and sham-ovariectomized rats $(P=\mathrm{ns})$ (Fig. $2 a-d)$.

Acute infusion of L-NAME or ANG II during chronic infusion of purified porcine $R L X$. The renal vasodilation and hyperfiltration in the RLX-treated rats observed on day 5 of infusion was completely abrogated by NO synthase inhibition (Table 1). To minimize the possibility that the convergence of renal function in the RLX and vehicle infused rats elicited by L-NAME was merely a consequence of nonspecific renal vasoconstriction, the experimental paradigm was repeated using another vasoconstrictor, ANG II. Rather than converging, ERPF and ERVR diverged even further during the acute infusion of ANG II (Table 2). Thus, these results were diametrically opposed to those obtained using the L-NAME. To emphasize the contrasting effects of L-NAME and ANG II on renal function and MAP in conscious rats chronically administered RLX, the percent change in MAP, GFR, ERPF, and ERVR from baseline is portrayed in Figs. 3 and 4. L-NAME produced a significantly greater rise in MAP and ERVR and reduction in GFR and ERPF in the RLX-treated compared with vehicle-infused rats $(P<0.05$ by ANOVA). In contrast, the percent increase in MAP and reduction in GFR elicited by ANG II was comparable in the RLX-treated and vehicle-infused rats, whereas the percent increase in ERVR and reduction in ERPF was markedly attenuated in the rats administered chronic $\operatorname{RLX}(P<0.001$ vs. vehicle by ANOVA). Another group of rats chronically treated with porcine RLX was administered Ringer's solution instead of L-NAME or ANG II, and thus served as time controls. Neither MAP nor any 


\section{Figure 1}

Effect of long-term infusion of purified porcine RLX, rhRLX, or vehicle on $(\boldsymbol{a})$ mean arterial pressure $(\boldsymbol{b})$ glomerular filtration rate $(\boldsymbol{c})$ effective renal plasma flow, and $(\boldsymbol{d})$ effective renal vascular resistance. The numbers in parentheses depict the number of rats tested. ${ }^{*} P<0.05$ vs. baseline and vehicle. $r h R L X$, recombinant human RLX; $R L X$, relaxin. a

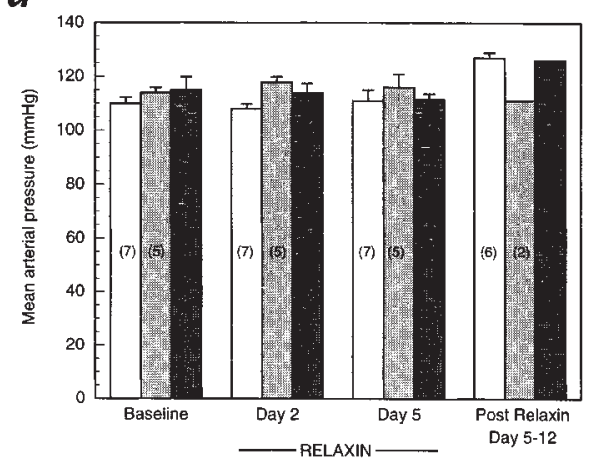

b

c

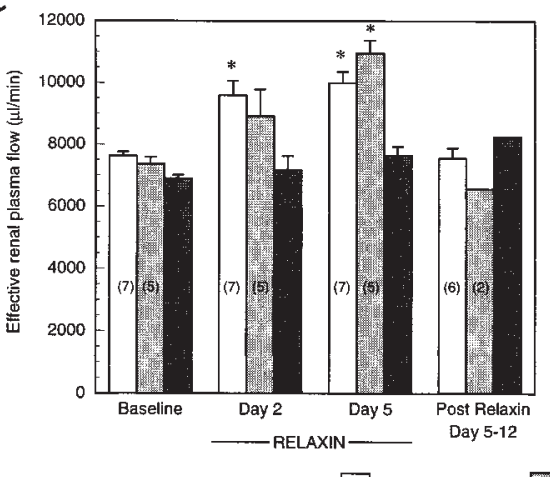

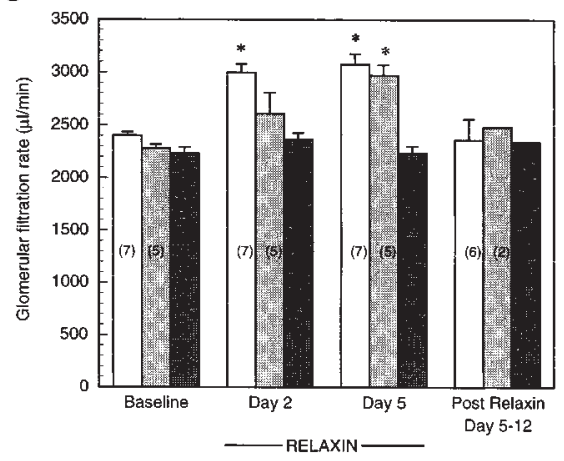

d

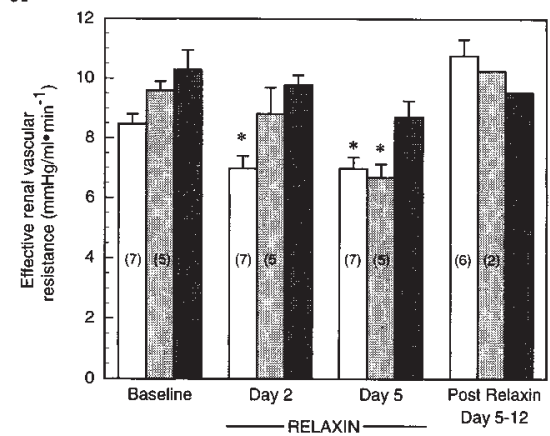

$\square$ Porcine Relaxin $\quad$ Human Relaxin $\quad$ Time Control

of the renal parameters were consistently altered over the approximately six hours of vehicle infusion (Table 3 ).

Acute administration of purified porcine $R L X$. A 2- $\mu \mathrm{g}$ bolus of purified porcine RLX followed by a sustained infusion of $4 \mu \mathrm{g} / \mathrm{h}$ for four hours did not affect either GFR or ERPF (Fig. 5, a-d).

Hematocrit and plasma osmolality. In the intact female rats chronically administered either purified porcine or rhRLX for the study of renal function, both the hematocrit and plasma osmolality showed significant decre- ments by day $2(P<0.05)$ (Table 4$)$.

Urinary excretion of $c G M P$ and $N O_{X}$. The 24-hour urinary excretion of cGMP and $\mathrm{NO}_{\mathrm{x}}$ were not significantly affected by the chronic infusion of porcine RLX at 4 $\mu \mathrm{g} / \mathrm{h}(P=\mathrm{ns}$ by ANOVA). In the same animals, urinary sodium excretion and water intake were significantly increased on days 2 and 5, respectively, of RLX infusion $(P<0.05$ vs. baseline and post-RLX) (Table 5).

Serum RLX. In 17 of the rats, we measured the serum concentration of the rhRLX that was infused by osmot-

Table 1

Effect of L-NAME on mean arterial pressure and renal function in conscious rats administered porcine RLX or Ringer's solution (vehicle) for 5 days ${ }^{A}$

\begin{tabular}{|c|c|c|c|c|c|}
\hline & \multirow[b]{2}{*}{ Baseline } & \multicolumn{4}{|c|}{ L-NAME } \\
\hline & & $60 \mathrm{~min}$ & $120 \mathrm{~min}$ & $180 \mathrm{~min}$ & $240 \mathrm{~min}$ \\
\hline \multicolumn{6}{|c|}{ Mean arterial pressure $(\mathrm{mmHg})$} \\
\hline RLX & $120 \pm 2$ & $135 \pm 5^{\mathrm{B}, \mathrm{C}}$ & $136 \pm 4^{\mathrm{B}, \mathrm{C}}$ & $136 \pm 4^{\mathrm{B}, \mathrm{C}}$ & $139 \pm 4^{B, C}$ \\
\hline Vehicle & $117 \pm 4$ & $122 \pm 4$ & $126 \pm 4$ & $129 \pm 4^{B}$ & $129 \pm 3^{B}$ \\
\hline \multicolumn{6}{|c|}{ Glomerular filtration rate $(\mu \mathrm{l} / \mathrm{min})$} \\
\hline RLX & $3,050 \pm 95^{c}$ & $2,776 \pm 142$ & $2,653 \pm 207$ & $2,498 \pm 113^{B}$ & $2,392 \pm 50^{B}$ \\
\hline Vehicle & $2,383 \pm 72$ & $2,419 \pm 170$ & $2,153 \pm 122$ & $2,350 \pm 63$ & $2,250 \pm 88$ \\
\hline \multicolumn{6}{|c|}{ Effective renal plasma flow $(\mu \mathrm{l} / \mathrm{min})$} \\
\hline RLX & $10,245 \pm 260^{C}$ & $7,520 \pm 481^{\mathrm{B}}$ & $7,030 \pm 419^{\mathrm{B}}$ & $6,530 \pm 270^{\mathrm{B}}$ & $5,973 \pm 195^{\mathrm{B}}$ \\
\hline Vehicle & $7,241 \pm 350$ & $7,301 \pm 385$ & $6,452 \pm 216^{B}$ & $6,595 \pm 507^{B}$ & $6,187 \pm 317^{B}$ \\
\hline \multicolumn{6}{|c|}{ Effective renal vascular resistance $(\mathrm{mmHg} / \mathrm{ml} / \mathrm{min})$} \\
\hline RLX & $6.96 \pm 0.23^{C}$ & $11.00 \pm 0.57^{\mathrm{B}}$ & $11.87 \pm 0.76^{\mathrm{B}}$ & $12.92 \pm 0.66^{\mathrm{B}}$ & $14.13 \pm 0.47^{\mathrm{B}}$ \\
\hline Vehicle & $10.48 \pm 0.29$ & $10.53 \pm 0.58$ & $12.28 \pm 0.58$ & $12.46 \pm 0.92$ & $12.64 \pm 0.73$ \\
\hline
\end{tabular}

AMean \pm SEM. L-NAME was infused intravenously at $2 \mu \mathrm{g} / \mathrm{min}$ and RLX at $4 \mu \mathrm{g} / \mathrm{h}$ by osmotic minipump subcutaneously. There were seven and six rats, respectively, in the RLX and vehicle groups. ${ }^{\mathrm{B}} P<0.05$ vs. baseline. ${ }^{C} P<0.05 \mathrm{RLX}$ vs. vehicle. $L-N A M E$, N ${ }^{\omega}$-nitro-L-arginine methyl ester; $R L X$, relaxin. 
Figure 2

Effect of 5-day administration of rhRLX to sham-ovariectomized and ovariectomized rats on $(\boldsymbol{a})$ mean arterial pressure $(\boldsymbol{b})$ glomerular filtration rate $(\boldsymbol{c})$ effective renal plasma flow, and $(\boldsymbol{d})$ effective renal vascular resistance. The numbers in parentheses depict the number of rats investigated. ${ }^{*} P<0.05$ vs. baseline. a

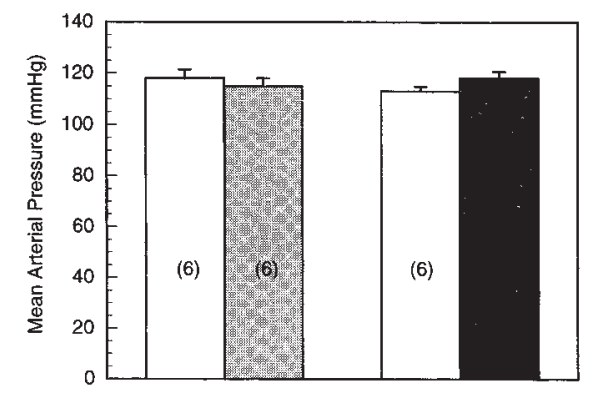

c

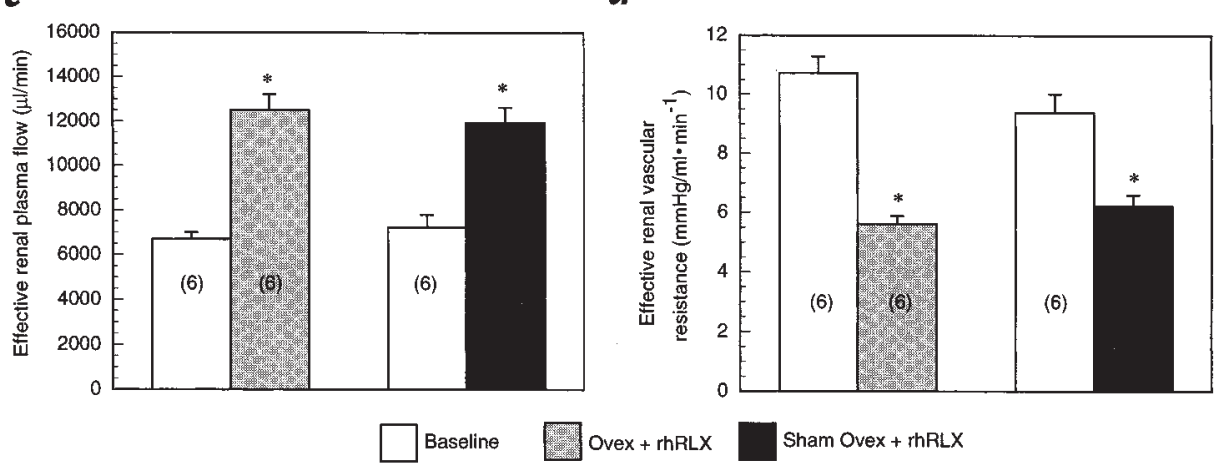

$\boldsymbol{b}$

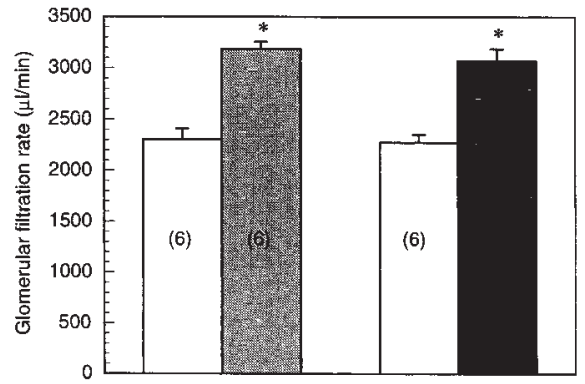

d ic minipump at $4 \mu \mathrm{g} / \mathrm{h}$ for five days. The mean \pm SEM was $28.1 \pm 4.8 \mathrm{ng} / \mathrm{ml}$.

\section{Discussion}

Our objective was to determine whether exogenous RLX would elicit renal vasodilation and hyperfiltration in conscious rats. The major findings were: $(a)$ chronic administration of either purified porcine or rhRLX markedly increased both GFR and ERPF; $(b)$ this increase was observed irrespective of the presence or absence of the ovaries; (c) L-NAME, a NO synthase inhibitor, completely abrogated the renal vasodilation and hyperfiltration elicited by chronic RLX infusion; and $(d)$ chronic administration of RLX markedly attenuated the renal vasoconstriction produced by ANG II.
Importantly, the renal vasodilatory effect of purified porcine RLX depended on the time course of administration. Neither GFR nor ERPF was altered during the acute infusion of purified porcine RLX ( $\leq 4$ hours), but both were markedly increased with more prolonged administration ( $\geq 48$ hours; Figs. 1 and 5). To exclude the remote possibility that this effect of porcine RLX on GFR and ERPF was secondary to some unidentified trace impurity in the porcine RLX preparation, we also administered rhRLX by subcutaneous osmotic minipump at the same rate of $4 \mu \mathrm{g} / \mathrm{h}$. The increase in both GFR and ERPF, as well as reciprocal decline in ERVR, were comparable between the two RLX preparations, although the time course may have been somewhat delayed for the rhRLX (Fig. 1). The magnitude of increase in GFR and

Table 2

Effect of ANG II on mean arterial pressure and renal function in conscious rats administered porcine RLX or Ringer's solution (vehicle) for 5 days ${ }^{A}$

\begin{tabular}{|c|c|c|c|c|c|}
\hline & \multirow[b]{2}{*}{ Baseline } & \multicolumn{4}{|c|}{ ANG II } \\
\hline & & $60 \mathrm{~min}$ & $120 \min$ & $180 \mathrm{~min}$ & $240 \mathrm{~min}$ \\
\hline \multicolumn{6}{|c|}{ Mean arterial pressure $(\mathrm{mmHg})$} \\
\hline RLX & $111 \pm 3$ & $130 \pm 5^{\mathrm{B}}$ & $142 \pm 4^{\mathrm{B}}$ & $143 \pm 5^{\mathrm{B}}$ & $146 \pm 6^{B}$ \\
\hline Vehicle & $112 \pm 5$ & $131 \pm 8$ & $140 \pm 9$ & $144 \pm 9^{B}$ & $145 \pm 9^{\mathrm{B}}$ \\
\hline \multicolumn{6}{|c|}{ Glomerular filtration rate $(\mu \mathrm{l} / \mathrm{min})$} \\
\hline RLX & $2,929 \pm 120^{C}$ & $2,980 \pm 219 c$ & $2,653 \pm 169 c$ & $2,578 \pm 169$ & $2,763 \pm 155^{C}$ \\
\hline Vehicle & $2,252 \pm 63$ & $2,339 \pm 123$ & $2,091 \pm 127$ & $2,297 \pm 59$ & $1,934 \pm 67$ \\
\hline \multicolumn{6}{|c|}{ Effective renal plasma flow $(\mu \mathrm{l} / \mathrm{min})$} \\
\hline RLX & $10,364 \pm 411^{c}$ & $8,926 \pm 478^{c}$ & $9,646 \pm 772^{C}$ & $7,738 \pm 386^{B, C}$ & $7,698 \pm 592^{\mathrm{B}, \mathrm{C}}$ \\
\hline Vehicle & $7,213 \pm 487$ & $5,689 \pm 453^{B}$ & $5,127 \pm 369^{\mathrm{B}}$ & $4,493 \pm 468^{B}$ & $4,053 \pm 118^{\mathrm{B}}$ \\
\hline \multicolumn{6}{|c|}{ Effective renal vascular resistance $(\mathrm{mmHg} / \mathrm{ml} / \mathrm{min})$} \\
\hline RLX & $6.23 \pm 0.41^{c}$ & $8.88 \pm 0.66^{\mathrm{B}, \mathrm{C}}$ & $9.24 \pm 1.03^{B, C}$ & $11.52 \pm 0.84^{\mathrm{B}, \mathrm{C}}$ & $11.84 \pm 1.08^{\mathrm{B}, \mathrm{C}}$ \\
\hline Vehicle & $9.03 \pm 0.54$ & $14.07 \pm 1.69$ & $16.59 \pm 1.76^{\mathrm{B}}$ & $20.53 \pm 2.31^{\mathrm{B}}$ & $21.81 \pm 1.56^{\mathrm{B}}$ \\
\hline
\end{tabular}

AMean \pm SEM. ANG II was infused intravenously at $3 \mathrm{ng} / \mathrm{min}$, and RLX at $4 \mu \mathrm{g} / \mathrm{h}$ by osmotic minipump subcutaneously. There were five rats each in the RLX and vehicle groups. ${ }^{B} P<0.05$ vs. baseline. ${ }^{C} P<0.05$ RLXvs. vehicle. ANG II, angiotensin II. 


\section{Figure 3}

The effect of L-NAME infusion in rats administered purified porcine $\operatorname{RLX}(n=7)$ or vehicle $(n=6)$ for 5 days. The means \pm SEM represent the percent change from baseline values for $(\boldsymbol{a})$ mean arterial pressure $(\boldsymbol{b})$ glomerular filtration rate (c) effective renal plasma flow, and (d) effective renal vascular resistance. L-NAME, $\mathrm{N}^{\omega}{ }^{\omega}$-nitro-L-arginine methyl ester.

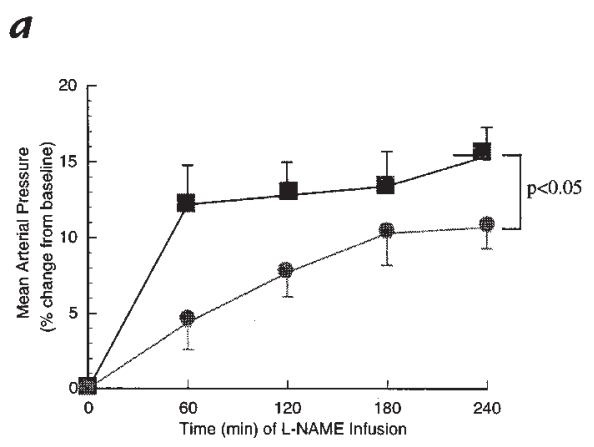

$\boldsymbol{b}$

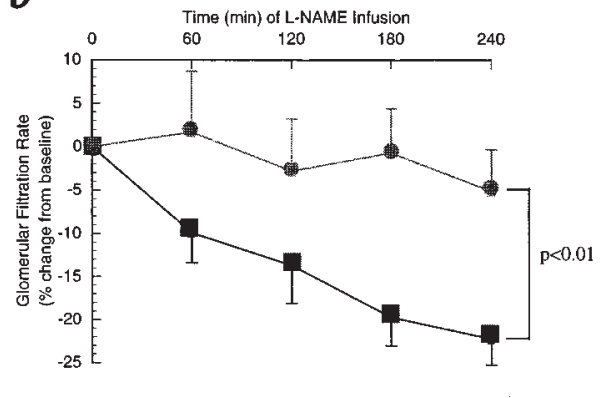

$c$

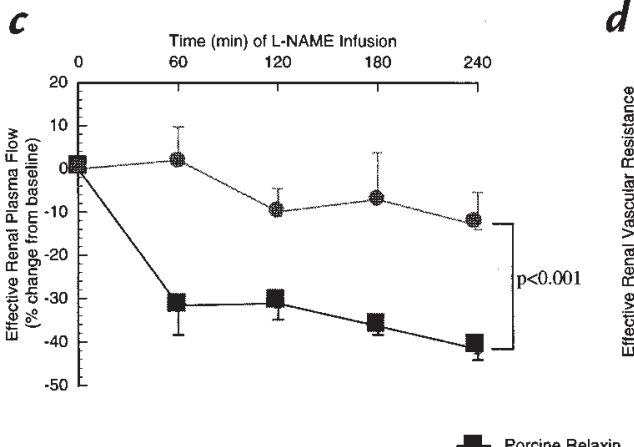

$d$

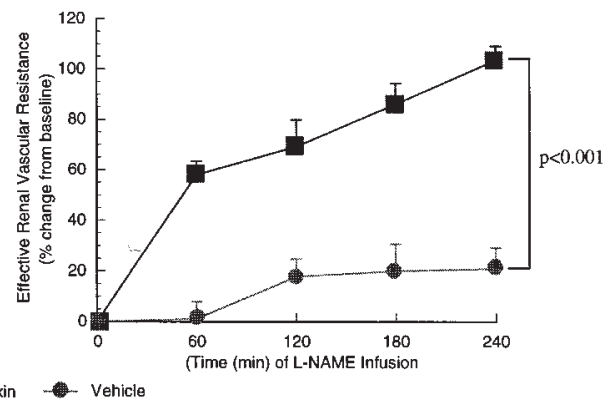

ERPF, as well as decline in ERVR, is similar to that observed during midgestation in rats ranging from $20 \%-40 \%(18,22)$. Importantly, time-control studies in which the vehicles for the two preparations of RLX were administered instead of the hormones showed relative stability of MAP and renal function (Fig. 1).

Based on the work of other investigators $(14,25)$ who administered purified porcine or rhRLX to rats and reported both the dosage given and circulating concentration reached, we were able to establish an approximate infusion rate of $4 \mu \mathrm{g} / \mathrm{h}$ that would produce serum levels comparable to those observed during gestational days $12-14$ in rats, i.e., $20-40 \mathrm{ng} / \mathrm{ml}$ (19). We chose levels of serum RLX to approximate values observed on gestational days 12-14 because both GFR and ERPF are maximal at this time in the pregnant rat $(18,22)$. When we measured the serum concentration of rhRLX on day 5 of chronic infusion, we indeed observed levels that were close to our target values (see Results). Of course, this approach to establishing a proper dose is only an approximation, because the relative potency of porcine and rhRLX compared with that of endogenous rat RLX, with respect to the renal circulation, is not known.

Some of the biological actions of RLX in the female reproductive tract depend on estrogen and/or progesterone, or are potentiated by the sex steroids (reviewed in ref. 1). However, this does not appear to be the case for the effect of RLX on the renal circulation (Fig. 2). The magnitude of change in renal function was comparable between ovariectomized and sham-ovariectomized rats, suggesting the lack of a permissive or potentiating role for estrogen or progesterone.

This study provides evidence that RLX exerts its effect on the renal circulation via NO (Table 1 and Fig. 3 ). Acute administration of inhibitors of NO synthase similarly abrogated renal vasodilation and hyperfiltration in conscious midterm pregnant rats (22). NO synthase inhibition, however, produced a significantly greater pressor effect in the RLX-treated rats, a finding that was not observed in midgestational rats (22). Differences in baroreflex control may account for the disparate effects of NO synthase blockade on blood pressure in RLX-treated rats compared with midterm pregnant rats, because the hypertensive response to L-

Table 3

Effect of Ringer's solution on mean arterial pressure and renal function in conscious rats administered porcine RLX for 5 days (time control)A

\begin{tabular}{|c|c|c|c|c|c|}
\hline & \multirow[b]{2}{*}{ Baseline } & \multicolumn{4}{|c|}{ Ringer's solution } \\
\hline & & $60 \mathrm{~min}$ & $120 \mathrm{~min}$ & $180 \mathrm{~min}$ & $240 \mathrm{~min}$ \\
\hline Mean arterial pressure $(\mathrm{mmHg})$ & $119 \pm 2$ & $123 \pm 3$ & $121 \pm 4$ & $122 \pm 4$ & $121 \pm 4$ \\
\hline Glomerular filtration rate $(\mu \mathrm{l} / \mathrm{min})$ & $2,997 \pm 98$ & $3,008 \pm 243$ & $3,086 \pm 143$ & $3,077 \pm 127$ & $3,223 \pm 121$ \\
\hline Effective renal plasma flow $(\mu \mathrm{l} / \mathrm{min})$ & $12,927 \pm 804$ & $12,630 \pm 1,140$ & $12,347 \pm 1,303$ & $12,353 \pm 1,266$ & $12,090 \pm 439$ \\
\hline Effective renal vascular resistance $(\mathrm{mmHg} / \mathrm{ml} / \mathrm{min})$ & $5.93 \pm 0.35$ & $6.19 \pm 0.48$ & $6.40 \pm 0.64$ & $6.52 \pm 0.68$ & $6.53 \pm 0.24$ \\
\hline
\end{tabular}

AMean \pm SEM, $n=4$ rats. Ringer's solution was infused at the same flow rate as L-NAME (Table 1 ) or ANG II (Table 2$)$ at $12.5 \mu \mathrm{l} / \mathrm{min}$. RLX was infused at $4 \mu \mathrm{g} / \mathrm{h}$ by osmotic minipump subcutaneously. 


\section{Figure 4}

The effect of ANG II infusion in rats administered purified porcine RLX or vehicle for 5 days. There were five rats in each treatment group. The means \pm SEM represent the percent change from baseline values for $(\boldsymbol{a})$ mean arterial pressure $(\boldsymbol{b})$ glomerular filtration rate $(\boldsymbol{c})$ effective renal plasma flow, and $(\boldsymbol{d})$ effective renal vascular resistance. ANG II, angiotensin II. $a$

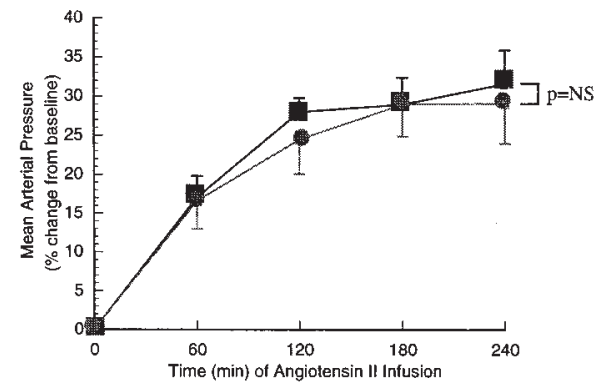

c

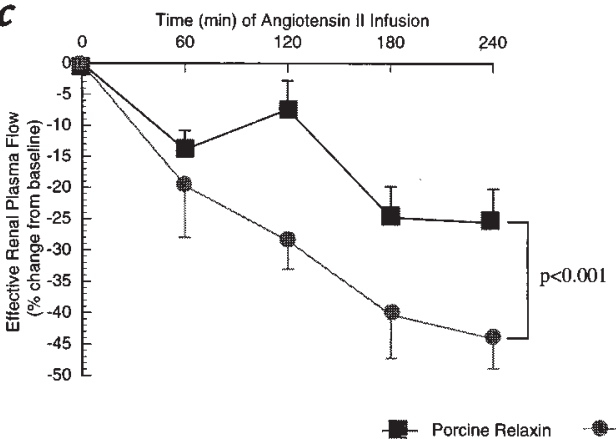

$b$

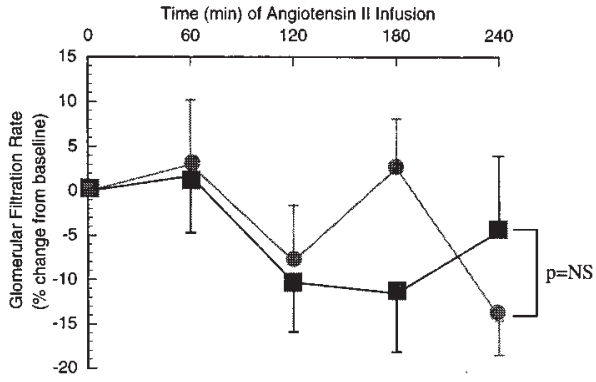

d

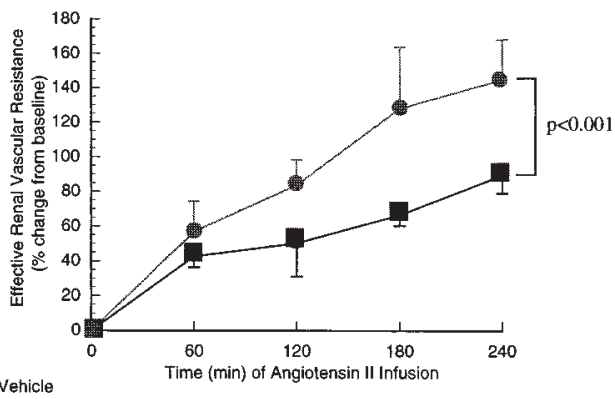

NAME was greater in pregnant rats subjected to ganglionic blockade (30). However, this possible explanation remains to be tested. A link between RLX and peripheral vasodilation, as well as NO, was reported previously. Specifically, chronic administration of rat RLX $(1.8 \mu \mathrm{g} /$ day $)$ reduced blood pressure and diminished the vasoconstrictor responses in the isolated, perfused mesenteric circulation of spontaneous hypertensive rats $(31,32)$. Moreover, perfusion of rat and guinea pig hearts in a Langendorff apparatus with porcine RLX significantly increased coronary blood flow via stimulation of $\mathrm{NO}(33)$.

To reduce the possibility that the abrogation of renal vasodilation and hyperfiltration in RLX-treated rats by NO synthase blockade was due merely to nonspecific renal vasoconstriction, we also administered ANG II. In contrast to LNAME, the renal vasoconstrictor effect of ANG II was attenuated rather than exaggerated in the rats administered RLX compared with those animals given vehicle, although the systemic pressor response to ANG II was unaffected by RLX administration (Table 2 and Fig. 4). In fact, similar attenuation of the renal vasoconstrictor action of ANG II was observed previously in midterm pregnant (22) and late gestational rats (34). In contrast to human pregnancy, reduced systemic pressor response to ANG II is not apparent until late gestation in the rat (34). Whether systemic vascular refractoriness develops earlier in rat pregnancy is unknown, because concurrent measurement of cardiac out-

Table 4 put and blood pressure has not been made during ANG II infusion in the species. Importantly, infusion of vehicle instead of either L-NAME or ANG II in RLX-treated rats showed relative stability of blood pressure and renal function over time (Table 3).

Previous investigators (14) demonstrated that chronic administration of RLX to ovariectomized rats reduced plasma osmolality and the osmotic threshold for arginine vasopressin release. Our work corroborates these findings, insofar as plasma osmolality fell by approximately $10-15 \mathrm{mOsmol} / \mathrm{kg}$ water on day 5 of purified porcine or rhRLX administration (Table 4).

Hematocrit and plasma osmolality of conscious rats chronically administered porcine RLX, rhRLX, or vehicle (time control) $)^{\mathrm{A}}$

$\begin{array}{llll}\text { Baseline Day 2 Day } 5 & \begin{array}{l}\text { Post-RLX } \\ \text { days 5-12 }\end{array} & \begin{array}{l}\text { Difference between } \\ \text { baseline and day } 5\end{array}\end{array}$

\begin{tabular}{lccccc} 
Hematocrit (\%) & & & \\
$\quad$ Porcine RLX & $42 \pm 1$ & $39 \pm 1^{\mathrm{B}}$ & $37 \pm 1^{\mathrm{B}, \mathrm{C}}$ & $40 \pm 1$ & $-4 \pm 1^{\mathrm{C}}$ \\
$\quad$ Vehicle & $40 \pm 3$ & $39 \pm 1$ & $40 \pm 1$ & 41 & $+2 \pm 3$ \\
$\quad$ rhRLX & $39 \pm 0$ & $37 \pm 1^{\mathrm{B}}$ & $37 \pm 1^{\mathrm{B}, \mathrm{C}}$ & 40 & $-2 \pm 1^{\mathrm{C}}$ \\
Plasma osmolality & & & & & \\
(mOsm/kg Ho) & & & & & \\
$\quad$ Porcine RLX & $301 \pm 2$ & $289 \pm 2^{\mathrm{B}, \mathrm{C}}$ & $287 \pm 2^{\mathrm{B}, \mathrm{C}}$ & $299 \pm 2$ & $-14 \pm 3^{\mathrm{C}}$ \\
$\quad$ Vehicle & $298 \pm 3$ & $297 \pm 2$ & $299 \pm 3$ & 299 & $0 \pm 2$ \\
$\quad$ rhRLX & $299 \pm 2$ & $291 \pm 3^{\mathrm{B}, \mathrm{C}}$ & $286 \pm 2^{\mathrm{B}, \mathrm{C}}$ & 302 & $-12 \pm 2^{\mathrm{C}}$ \\
\hline
\end{tabular}

${ }^{A}$ Mean \pm SEM. RLX was infused at $4 \mu \mathrm{g} / \mathrm{h}$ by osmotic minipump subcutaneously. There were seven, four, and five rats, respectively, in the porcine RLX, vehicle, and rhRLX groups at baseline and at days 2 and 5 of treatment. During the post-RLX period, six, two, and two rats were studied in the porcine RLX, vehicle, and rhRLX groups. Two of the rats in the vehicle group received Ringer's solution (vehicle for porcine RLX), and two received $20 \mathrm{mM}$ sodium acetate ( $\mathrm{pH}$ 5.0) (vehicle for rhRLX). The results were not different; therefore, the data were combined. ${ }^{\mathrm{B}} P<0.05$ vs. baseline. ${ }^{C} P<0.05$ RLX vs. vehicle. rhRLX, recombinant human relaxin. 


\section{Figure 5}

Effect of short-term infusion of purified porcine RLX on (a) mean arterial pressure $(\boldsymbol{b})$ glomerular filtration rate (c) effective renal plasma flow, and (d) effective renal vascular resistance. $n=4$ rats. a
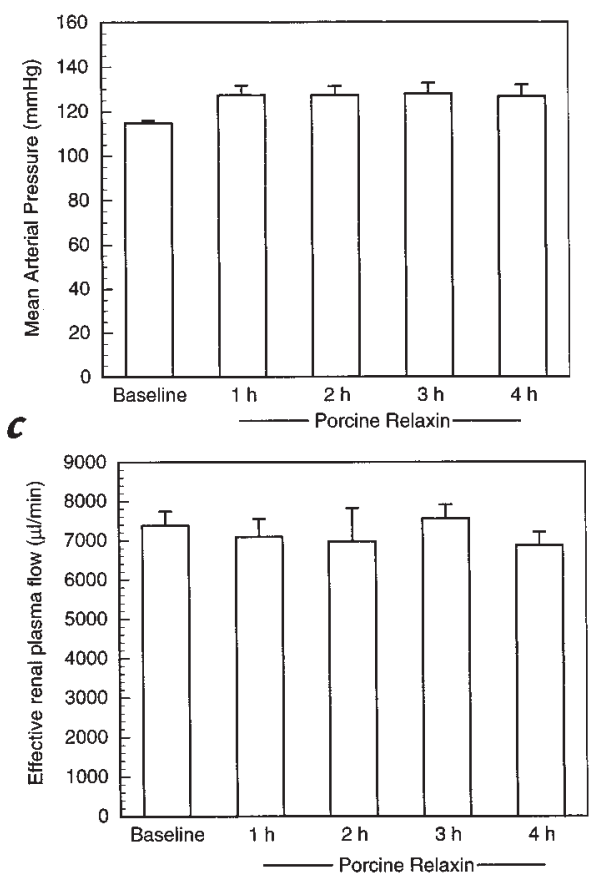

$b$
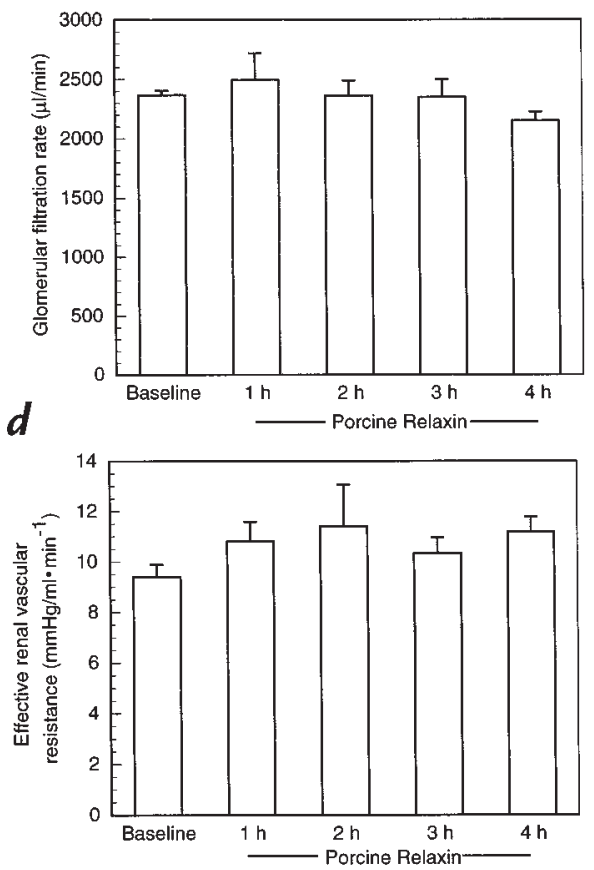

This event was associated with a significant reduction in hematocrit, suggesting plasma volume expansion. In metabolism cage studies (Table 5), water intake was also noted to be significantly increased on day 5 of hormone administration, which is consistent with a previous report demonstrating that endogenous RLX increased water intake in rats during the light period in the second half of gestation (35). Because a decline in the plasma osmolality and osmotic threshold for arginine vasopressin release and water appetite are maternal adaptations in pregnancy (13-17), it is tempting to speculate that RLX contributes to these responses, especially since they roughly correspond with the appearance of the hormone in the circulation of both rats and humans $(1,19)$. Thus, circulating RLX may directly mediate these osmoregulatory changes in the central nervous system via the circumventricular organs or indirectly by reducing total peripheral vascular resistance, thus effecting nonosmotic release of arginine vasopressin and alteration of the threshold for thirst. Alternatively, these osmoregulatory changes may be mediated by local production of RLX and its action on receptors in the central nervous system (36).

Pregnancy in rats and women is accompanied by a marked increase in urinary excretion and/or plasma level of cGMP (reviewed in ref. 37). The chronic administration of purified porcine RLX decidedly failed to mimic this finding (Table 5), despite its renal vasodilatory effect mediated by NO, and presumably cGMP, the second messenger of NO. The paral- lel increase of cGMP and the NO metabolites, nitrate and nitrite, in both the plasma and urine of gravid rats may result mainly from the expression of the inducible form of NO synthase in other tissues besides the vasculature, thereby having little or no relation to the vasodilatory changes of pregnancy (38). In other words, the profound renal vasodilatory effects of pregnancy and of RLX infusion in nonpregnant rats both may be mediated by increased vascular production of $\mathrm{NO}$ by the endothelial isoform of the enzyme, but the increase in NO generation is relatively small and, consequently, does not significantly impact on the urinary excretion of nitrate and cGMP, which is already substantial.

In summary, this study demonstrates that prolonged administration of RLX is vasodilatory in the renal circulation of conscious rats. This effect is independent of other ovarian hormones but dependent on NO. Because the impact of exogenous RLX on the renal circulation mimics that of pregnancy, a role for endogenous RLX in the control of renal vasodilation and hyperfiltration during pregnancy warrants investigation.
Table 5

24-h urinary excretion of CGMP and $\mathrm{NO}_{x}$ in conscious rats administered porcine RLX for 5 days ${ }^{\mathrm{A}}$

\begin{tabular}{|c|c|c|c|c|}
\hline & Baseline & Day 2 & Day 5 & Post-RLX days 4- \\
\hline Food intake (g) & $13.2 \pm 1.0$ & $15.5 \pm 1.5$ & $16.7 \pm 1.0$ & $15.3 \pm 0.5$ \\
\hline Water intake $(\mathrm{ml})$ & $19.1 \pm 2.7$ & $20.1 \pm 3.4$ & $23.6 \pm 3.3^{\mathrm{B}}$ & $19.1 \pm 2.6$ \\
\hline Urine output ( $\mathrm{ml} / 24 \mathrm{~h})$ & $9.1 \pm 1.7$ & $11.4 \pm 2.2$ & $8.8 \pm 0.9$ & $9.1 \pm 1.1$ \\
\hline Urinary sodium excretion $(\mu \mathrm{Eq} / 24 \mathrm{~h})$ & $438.8 \pm 34.4$ & $800.2 \pm 77.2^{\mathrm{B}}$ & $477.8 \pm 57.9$ & $480.69 \pm 54.4$ \\
\hline Urinary cGMP excretion ( $\mathrm{nmol} / 24 \mathrm{~h})$ & $32.2 \pm 1.9$ & $26.5 \pm 2.5$ & $28.6 \pm 3.8$ & $32.7 \pm 3.8$ \\
\hline Urinary $\mathrm{NO}_{x}$ excretion $(\mu \mathrm{mol} / 24 \mathrm{~h})$ & $4.8 \pm 0.5$ & $6.0 \pm 1.0$ & $5.9 \pm 0.7$ & $6.1 \pm 0.9$ \\
\hline
\end{tabular}

${ }^{A}$ Mean \pm SEM, $n=6$ rats. RLX was infused at $4 \mu \mathrm{g} / \mathrm{h}$ by osmotic minipump subcutaneously. ${ }^{\mathrm{B} P}<0.05$ vs. baseline and post-RLX administration. $\mathrm{NO}_{X}$, nitrate + nitrite. 


\section{Acknowledgments}

We thank Beverly Grove of Connectics for performing the human relaxin immunoassay, and Elaine Unemori, also of Connectics, for generously providing the recombinant human relaxin 2 . The expert technical assistance of Mike Bodenheimer and Laurie Kerchner in all aspects of the metabolism cage studies is also gratefully acknowledged. Finally, we thank Betty Skippers for assisting with the statistical analyses, and Sue Davis for superb secretarial support. This work was supported by the National Institutes of Health grants R01 HD-30325 and RCDA K04 HD-01098.

1. Sherwood, O.D. 1994. Relaxin. In The physiology of reproduction. 2nd ed. E. Knobil, J.D. Neill, G.S Greenwald, C.L. Markert, and D.W. Pfaff, editors. Raven Press. New York, NY. 861-1009.

2. Gilson, G.J., Mosher, M.D., and Conrad, K.P. 1992. Systemic hemodynamics and oxygen transport during pregnancy in chronically instrumented, conscious rats. Am. J. Physiol. 263:H1911-H1918.

3. Conrad, K.P., and Lindheimer, M. 1999. Renal and cardiovascular changes in normal pregnancy and preeclampsia. In Chesley's bypertensive disorders in pregnancy. 2nd ed. M.D. Lindheimer, F.G. Cunningham, and J.M. Roberts, editors. Appleton \& Lange. Stamford, CT. 263-326.

4. Stewart, D.R., et al. 1990. Relaxin in the peri-implantation period. J. Clin. Endocrinol. Metab. 70:1771-1773.

5. Johnson, M.R., Carter, G., Grint, C., and Lightman, S.L. 1993. Relationship between ovarian steroids, gonadotrophins and relaxin during the menstrual cycle. Acta. Endocrinol. 129:121-125.

6. Wreje, U., Kristiansson, P., Åberg, G., Byström, B., and von Schoultz, B. 1995. Serum levels of relaxin during the menstrual cycle and oral contraceptive use. Gynecol. Obstet. Invest. 39:197-200.

7. Davison, J.M., and Noble, M.C.B. 1981. Serial changes in 24-hour creatinine clearance during normal menstrual cycles and the first trimester of pregnancy. Br. J. Obstet. Gynaecol. 88:10-17.

8. Paaby, P., et al. 1987. Endogenous overnight creatinine clearance compared with ${ }^{51} \mathrm{Cr}$-EDTA clearance during the menstrual cycle. Acta Med. Scand. 222:281-284

9. Paaby, P., Moller-Petersen, J., Larsen, C.E., and Raffn, K.1987. Endogenous overnight creatinine clearance, serum $\beta_{2}$-microglobulin and serum water during the menstrual cycle. Acta Med. Scand. 221:191-197.

10. Brochner-Mortensen, J., et al. 1987. Renal haemodynamics and extracellular homeostasis during the menstrual cycle. Scand J. Clin. Lab. Invest. 47:829-835.

11. Van Beek, E., et al. 1996. Peripheral haemodynamics and renal function in relation to the menstrual cycle. Clin. Sci. 91:163-168.

12. Chapman, A.B., et al. 1997. Systemic and renal hemodynamic changes in the luteal phase of the menstrual cycle mimic early pregnancy. Am.J. Physiol. 273:F777-F782.

13. Davison, J.M., Vallotton, M.B., and Lindheimer, M.D. 1981. Plasma osmolality and urinary concentration and dilution during and after pregnancy: evidence that lateral recumbency inhibits maximal urinary concentrating ability. Br. J. Obstet. Gynaecol. 88:472-479.

14. Weisinger, R.S., Burns, P., Eddie, L.W., Wintour, E.M. 1993. Relaxin alters the plasma osmolality-arginine vasopressin relationship in the rat. $J$. Endocrinol. 137:505-510.

15. Davison, J.M., Shiells, E.A., Philips, P.R., and Lindheimer, M.D. 1988. Serial evaluation of vasopressin release and thirst in human pregnancy. $J$. Clin. Invest. 81:798-806.

16. Lindheimer, M.D., Barron, W.M., and Davison, J.M. 1989. Osmoregulation of thirst and vasopressin release in pregnancy. Am. J. Physiol. 257:F159-F169.
17. Lindheimer, M.D., and Davison, J.M. 1995. Osmoregulation, the secretion of arginine vasopressin and its metabolism during pregnancy. Eur. J. Endocrinol. 132:133-143.

18. Conrad, K.P. 1984. Renal hemodynamics during pregnancy in chronically catheterized, conscious rats. Kidney Int. 26:24-29.

19. Sherwood, O.D., Crnekovic, V.E., Gordon, W.L., and Rutherford, J.E. 1980. Radioimmunoassay of relaxin throughout pregnancy and during parturition in the rat. Endocrinology. 107:691-698.

20. Atherton, J.C., Bu'lock, D., and Pirie, S.C. 1982. The effect of pseudopregnancy on glomerular filtration rate and salt and water reabsorption in the rat. J. Physiol. 324:11-20.

21. Baylis, C. 1982. Glomerular ultrafiltration in the pseudopregnant rat. Am. J. Physiol. 234:F300-F305.

22. Danielson, L.A., and Conrad, K.P. 1995. Acute blockade of nitric oxide synthase inhibits renal vasodilation and hyperfiltration during pregnancy in chronically instrumented conscious rats. J. Clin. Invest. 96:482-490.

23. Conrad, K.P., Gandley, R.E., Ogawa, T., Nakanishi, S., and Danielson, L.A. Endothelin mediates renal vasodilation and hyperfiltration during pregnancy in chronically instrumented conscious rats. Am. J. Physiol. In press.

24. Gandley, R.E., Conrad, K.P., and McLaughlin, M.K. 1998. Endothelin and nitric oxide regulate myogenic behavior in the renal resistance vasculature at mid-pregnancy in the rats. FASEB J. 12:A99. (Abstr.)

25. Burger, L.L., and Sherwood, O.D. 1995. Evidence that cellular proliferation contributes to relaxin-induced growth of both the vagina and the cervix in the pregnant rat. Endocrinology. 136:4820-4826.

26. Conrad, K.P., and Vernier, K.A. 1989. Plasma level, urinary excretion and metabolic production of cGMP during gestation in rats. Am. J. Physiol. 257:R847-R853.

27. Unemori, E.N., et al. 1996. Relaxin induces an extracellular matrixdegrading phenotype in human lung fibroblasts in vitro and inhibits lung fibrosis in a murine model in vivo. J. Clin. Invest. 98:2739-2745.

28. Sherwood, C.D., and O'Byrne, E.M. 1974. Purification and characterization of porcine relaxin. Arch. Biochem. Biophys. 160:185-196.

29. Zar, J.H. 1984. Biostatistical analysis. Prentice Hall. Englewood Cliffs, NJ.

30. Nathan, L., Cuevas, J., and Chaudhuri, G. 1995. The role of nitric oxide in the altered vascular reactivity of pregnancy in the rat. Br. J. Pharmacol. 114:955-960.

31. St-Louis, J., and Massicotte, G. 1985. Chronic decrease of blood pressure by rat relaxin in spontaneously hypertensive rats. Life Sci. 37:1351-1357.

32. Massicotte, G., Parent, A., and St.-Louis, J. 1989. Blunted responses to vasoconstrictors in mesenteric vasculature but not in portal vein of spontaneously hypertensive rats treated with relaxin. Proc. Soc. Exp. Biol. Med. 190:254-259.

33. Bani-Sacchi, T., Bigazzi, M., Bani, D., Mannaioni, P.F., and Masini, E. 1995. Relaxin-induced increased coronary flow through stimulation of nitric oxide production. Br. J. Pharmacol. 116:1589-1594.

34. Conrad, K.P., and Colpoys, M.C. 1986. Evidence against the hypothesis that prostaglandins are the vasodepressor agents of pregnancy. Serial studies in chronically instrumented, conscious rats. J. Clin. Invest. 77:236-245.

35. Zhao, S., Malmgren, C.H., Shanks, R.D., and Sherwood, O.D. 1995. Monoclonal antibodies specific for rat relaxin. VIII. Passive immunization with monoclonal antibodies throughout the second half of pregnancy reduces water consumption in rats. Endocrinology. 136:1892-1897.

36. Summerlee, A.J.S., Hornsby, D.J., and Ramsey, D.G. 1998. The dipsogenic effects of rat relaxin: the effect of photoperiod and the potential role of relaxin on drinking in pregnancy. Endocrinology. 139:2322-2328.

37. Sladek, S.M., Magness, R.R., and Conrad, K.P. 1997. Nitric oxide and pregnancy. Am. J. Physiol. 272:R441-R463.

38. Suto, T., Lateef, A., and Baylis, C. 1997. Effect of different nitric oxide synthesis (NOS) inhibitors on NO production and blood pressure (BP) in pregnancy $(\mathrm{P})$. Hypertension in Pregnancy. 16:68. (Abstr.) 